\title{
Unidad de Acción Comprometida: una propuesta de solución ante el problema universitario del servicio a la sociedad
}

\section{Unit of Committed Action: A proposal for a solution for the university problem of providing a service to the society}

\author{
Vicente Manzano-Arrondo* y Enrique Suárez García** \\ Fecha de recepción: 17-09-2014 - Fecha de aceptación: 8-11-2014 \\ Hábitat y Sociedad (ISSN 2173-125X), n. ${ }^{\circ}$, noviembre de 2015, pp. 147-165.
}

\begin{abstract}
Summary
The three traditionally assumed functions of the university (teaching, research, and knowledge transfer) have been suffering problems of mismatching (applications with different directions for each function), imbalance (some functions being more relevant than others), and of orientation (difficulties orienting work towards a service to society). Currently, market orientation clashes with 3covers university activity in its three commitments, thereby generating specific problems. This document analyses this situation and proposes Units of Committed Action as a contribution towards the set of solution strategies that are being generated to guide the university towards the service of common good, adjust the three commitments, and to foment a balance in each of their weights. The Units of Committed Action work along the same lines as Participatory Action Research and those of Service Learning, whose particularities are also addressed here. Finally, a specific application is presented in a neighbourhood catalogued as a zone in need of social transformation.
\end{abstract}

\section{Key words}

Unit of Committed Action, Action research, Service learning, Mission of the University

\begin{abstract}
Resumen
Las tres funciones tradicionalmente asumidas de la universidad (docencia, investigación y extensión) han venido sufriendo problemas de desajuste (concreciones con direcciones distintas para cada función), desequilibrio (mayor relevancia de unas funciones sobre otras) y orientación (dificultades para orientar el trabajo hacia el servicio a la sociedad). En estos momentos, la orientación economicista baña la actividad universitaria en sus tres cometidos, generando algunos problemas específicos. Este documento analiza esta situación y propone las Unidades de Acción Comprometida como una aportación al conjunto de estrategias de solución que se están generando para orientar la universidad al servicio del bien común, ajustar los tres cometidos en ese sentido y fomentar un equilibrio entre el peso de cada uno de ellos. Las Unidades de Acción Comprometidas trabajan en la misma línea que los modelos de Investigación-Acción Participativa y de Aprendizaje-Servicio, cuyas particularidades son también abordadas. Finalmente, se expone una aplicación concreta en un barrio de la ciudad catalogado como zona necesitada de transformación social.
\end{abstract}

\section{Palabras clave}

Unidad de acción comprometida, Investigación-acción, Aprendizaje-servicio, Misión de la Universidad

\footnotetext{
* Profesor titular de Metodología de las Ciencias del Comportamiento. Universidad de Sevilla. Departamento de Psicología Experimental. Email: vmanzano@us.es.

** Activista social y pregraduado en psicología por la Universidad de Sevilla. Comunidad de Investigación, Acción y Desarrollo para superar la Opresión (CIADO). Email: kike@equipociado.org.
} 


\section{Introducción}

Siguiendo un amplio consenso, la misión de la universidad se articula mediante tres grandes funciones o cometidos: la docencia (o educación, formación, enseñanza), la investigación (o innovación, construcción y gestión del conocimiento) y la extensión (o servicio a la sociedad). Los tres frentes han sido y siguen siendo objeto de una gran atención (por ejemplo, Clark, 1983; Ellacuria, 1999; Manzano-Arrondo y Torrego, 2009; Manzano-Arrondo, 2012; Morales, 2004). Este esfuerzo analítico, no obstante, no ha derivado en la solución de algunos problemas ya clásicos en torno a la concreción práctica de los tres cometidos.

En primer lugar, se observa un fuerte desajuste entre las tres funciones. Desde la perspectiva de un ajuste perfecto, un profesor universitario, por ejemplo, impartiría clases sobre el mismo tema del que investiga, a la vez que aplica estos contenidos en tareas específicas de servicio a la sociedad. En segundo lugar, existe un claro desequilibrio entre las tres funciones. Resulta cada vez más evidente, por ejemplo, el protagonismo del cometido investigador frente al docente, a la vez que de este sobre el de extensión. Y, por último, la orientación con que la universidad articula los tres frentes sufre un problema de definición que somete a la institución a una fuerte crisis (Sousa, 2006), que tiende a resolverse a favor de una clara perspectiva mercantil (Greenwood, 2012).

Este trabajo plantea abordar las condiciones que generan, mantienen y refuerzan los tres problemas mencionados (desajuste, desequilibrio y orientación mercantil), y propone una posible solución: las Unidades de Acción Comprometida (UAC). Con esta motivación, el documento define las UAC a través de antecedentes bien conocidos, a la vez que describe una aplicación concreta en un contexto de barrio urbano.

\section{Desajuste}

La actitud es un concepto ampliamente utilizado en las ciencias del comportamiento, cuya versión más extendida implica considerar tres componentes (Dawes, 1975; Morales, 2000): cognitivo (creencias, pensamientos), emotivo (sentimientos, emociones) y conativo (conductas motoras, observables). En otros términos, suele describirse como un modo coherente de pensar, sentir y hacer. Así, por ejemplo, la actitud racista implica creer en la superioridad e inferioridad de las personas en función de su etnia, sentir desprecio por las que se consideran inferiores y admiración por las consideradas superiores, y conducirse en la práctica cotidiana de forma coherente con tales creencias y emociones. Cuando una persona no se comporta de forma coherente, se provoca lo que Festinger (1957) denominaba disonancia cognitiva, caracterizada al menos por un estado desagradable o incómodo del ánimo, que tiende a resolverse habitualmente de forma imperfecta mediante diversos recursos como son las racionalizaciones (argumentaciones que intentan forzar la coherencia).

La universidad vive un estado similar a la disonancia cognitiva debido al modo deslavazado con que se concreta la práctica de sus tres funciones básicas.

Desde los primeros momentos históricos de lo que llamamos hoy universidad, se consideraba a esta una comunidad de maestros y apren- 
dices en torno al conocimiento (Andrés y Manzano, 2004), siguiendo el modelo general de adquisición de un oficio en la Edad Media. La comunidad se organizaba en las funciones de construcción y transmisión de conocimiento, es decir, en los cometidos de investigación y docencia, sin que la extensión tuviera una entidad concreta por sí misma. Este nacimiento ha constituido la impronta del imaginario académico, de tal forma que un profesor universitario ha de encarnar la pasión por el conocimiento, la adecuación a los estándares de investigación científica, la tendencia a comunicar, la erudición y el interés por la mejora pedagógica (Corlett, 2005), a lo que se añade con frecuencia y fuerza la defensa de la libertad académica (Cheng, 2011; Derrida, 2002) y, en menor medida, la pasión docente, es decir, por transmitir el conocimiento (Cunningham, 2007). No obstante, en entrevistas realizadas a numerosos miembros del profesorado anglosajón, Macfarlane (2011) encontró que las características más sobresalientes en las respuestas fueron relativas únicamente a la investigación y a la publicación científica.

La actividad docente permanece presente en todas las legislaciones en materia de instituciones de educación superior, en las cartas fundacionales, en los discursos institucionales... pero no es raro observar su ausencia en descripciones sobre el quehacer relevante de un miembro del profesorado universitario. Peor papel le toca a la extensión universitaria o servicio a la sociedad, habitualmente relegada a una responsabilidad general institucionalizada, pero fuera de la competencia de sus miembros. Hay que entender que el imaginario académico no se caracteriza por una especial preocupación en torno a la utilidad del progreso científico o del conocimiento. La utilidad de este quehacer tal vez pueda ser considerada como efecto secundario cuya gestión podría entrar en la competencia de emprendedores empresariales, profesionales de la política o personal técnico de las administraciones. Martínez (2012) fundamenta este hábito en el desprecio histórico que el intelectualismo ha sentido por las concreciones cotidianas, en contraste con un saber popular y cercano del que ha procurado separarse.

Esta concepción es quizá fácil de entender al observar el modo en que la institución otorga el rango de miembro académico. Por lo general es una necesidad docente la que justifica la contratación o la convocatoria de oposiciones para la entrada de nuevo profesorado. Las necesidades docentes pueden encontrarse dispersas y ser de naturaleza temporal. Quien accede no tiene por qué contar con una alta motivación para esos contenidos concretos. En su lugar, tal vez aspire a beneficiarse de la relativa movilidad intradepartamental y dedicar un día su actividad docente a una materia que realmente le apasiona. El ingreso en los asuntos de investigación consiste habitualmente en subir a uno de los barcos que ya están en marcha, y mejor cuanto más afianzado y productivo sea el grupo en el que se ingresa. Los motivos de investigación y los de docencia no siguen las mismas lógicas ni prácticas de acceso, por lo que es casi una suerte que los miembros del profesorado investiguen y enseñen sobre los mismos temas precisos. Cuando esto ocurre se debe más a un interés específico por conseguirlo a lo largo de los años, que por causa de los modos de ingreso.

Peor suerte corre la extensión. Si bien las necesidades docentes justifican la contratación de nuevo profesorado, es su productividad investigadora lo que robustece el currículum que permite hacer carrera y acceder a recursos y oportunidades (Cerezo, 2007). Aun más disonante resulta que ni en las condiciones de ingreso, ni en los procesos poste- 
riores de refuerzo exista mención alguna a tareas que impliquen servicio universitario a la comunidad (García-Quero, 2014). Este apartado ha sido asumido por la institución como institución, o bien por miembros universitarios habitualmente de forma individual y desconectada con el resto de las tareas académicas (Manzano-Arrondo, 2012). De este modo, no solo la extensión se encuentra sensiblemente empequeñecida, sino que la disonancia se expresa fundamentalmente en la circunstancia de que la práctica de la extensión nada tiene que ver con los motivos que se abordan en la docencia ni en la investigación.

\section{Desequilibrio}

Para Prilleltensky (2004), el bienestar es la sinergia balanceada en la atención a tres tipos de necesidades básicas: personales (nivel interno), relacionales (nivel interpersonal) y colectivas (nivel societal). Las tres facetas se encuentran íntimamente relacionadas. Así, para que alguien pueda disfrutar a nivel personal de un buen estado de salud, requiere acceso a agua potable o suficiente atención comunitaria. Si uno de los tres componentes se encuentra muy desarrollado oprime al resto. Si uno se encuentra desatendido, imposibilita la sinergia. En otras palabras, las necesidades y su dinámicas tienen base subjetiva tanto como material y política. Este modelo de equilibrio está inspirado en otro, propuesto por Miller (citado por Prilleltensky, 2008), en el que la justicia es el equilibrio o sinergia balanceada de tres elementos: la igualdad, el mérito y la satisfacción de las necesidades. De ese modo, cuando uno de los tres elementos es priorizado sobre los otros dos, o es desatendido, la justicia se debilita y decrece.

Los modelos de Miller y Prilleltensky constituyen buenas inspiraciones para concebir el modo en que la universidad debería abordar su misión mediante la articulación de las tres funciones básicas: ha de constituir una buena sinergia balanceada. Si uno de los elementos prima sobre el resto o es desatendido, el conjunto queda lastimado. Este aspecto es abordado específicamente por Siegel (1984) quien denuncia que el conocimiento superior es insuficiente para realizar una labor universitaria competente, y es necesario combinarlo con una educación superior y, literalmente, una ayuda superior. Esta combinación equilibrada justifica la potencia que podría estar en manos de la institución universitaria.

Sin embargo, como ya hemos indicado en el apartado anterior, ocurren los dos fenómenos indicados para el desequilibrio: refuerzo de unos, descuido de otros. La investigación es crecientemente priorizada, mientras que la extensión es mantenida a un nivel de efecto secundario. Este comportamiento no solo se asienta, como ya ha sido señalado, en el imaginario académico, sino que viene fortalecido sensiblemente por las reformas en materia universitaria, herederas tanto de la nueva gestión pública como de la concreción de los índices de impacto.

El modelo de la Nueva Gestión Pública (NPM, del original inglés New Public Management), se fundamenta en movimientos propuestos desde el mundo anglosajón, principalmente experimentados en Australia, Nueva Zelanda (Christensen, 2011) y Chile (Spinoza, 2008) desde los primeros años de la década de los ochenta. Consiste básicamente en aplicar el modelo de gestión empresarial a las administraciones públicas, priorizando la competitividad, la eficiencia y la productivi- 
dad y estableciendo una orientación hacia el consumidor o cliente. Se encuentra perfectamente en sintonía con el progresivo protagonismo de la privatización de un mayor número de esferas de la cotidianidad (Guillén, 2003) que venía desarrollándose desde el inicio del capitalismo hasta nuestros días.

La perspectiva academicista es susceptible de ser valorada desde un punto de vista cualitativo, centrado en las condiciones de trabajo con el conocimiento, sea desde labores de investigación o de docencia. Sin embargo, la perspectiva mercantil que subyace al modelo NPM, exige un abordaje cuantitativo, donde prima la contabilidad de unidades de producción y la evaluación de la calidad de tales unidades recurriendo a mecanismos uniformadores, es decir, aplicables a una amplia diversidad de procedimientos, cometidos y resultados. El énfasis por la productividad es uno de los fundamentos más sólidos para comprender la actual focalización hacia el número de publicaciones en revistas con índice de impacto. Hay que tener en cuenta que la calidad docente es menos susceptible de ser reducida a un índice meramente cuantitativo, mientras que esta circunstancia sí explica el éxito de las estrategias de medición del impacto del quehacer universitario a través del número de unidades publicadas según los estándares de referencia (Cunningham, 2007).

Uno de los elementos más claramente definitorios del ámbito empresarial es el criterio de la maximización de los beneficios. Desde los primeros momentos de implantación del NPM sobre la universidad, este criterio se ha vivido en términos de mal encuadre, de objetivo impropio de una institución de educación (Worthington y Higgs, 2011). No obstante, el desencuadre ha sido simbólicamente solucionado a través del concepto del sistema de valor de prestigio (PVS, por su original inglés Prestige Value System). El marco de referencia que suministra PVS permite aplicar el objetivo de la maximización en el contexto universitario, concretándolo sobre el prestigio del personal docente e investigador (Morrison et al., 2011). El objetivo de la institución y de sus miembros puede ser entonces concebido como de maximización del prestigio, operado a través de la consecución de fondos para investigación y de publicaciones en revistas científicas de impacto.

El conjunto de estas dinámicas fortalecen el protagonismo de la función investigadora y, muy especialmente, la medida de productividad y prestigio a través del número de publicaciones realizadas. A su vez, el conjunto de las publicaciones realizadas o realizables a considerar es sensiblemente reducido al contemplar únicamente aquellas que cuentan con un índice de calidad organizado coherentemente desde la misma perspectiva de productividad cuantitativa: las publicaciones contenidas en el sistema Journal Citation Reports (JCR) de la empresa Thomson Reuters. Las sucesivas reformas universitarias van incrementando la importancia de este sistema de medición (Quinn, 2012), incrementando las consecuencias sobre las condiciones laborales, las posibilidades de progreso académico y el sistema de valor de prestigio del profesorado universitario y de las instituciones de educación superior (Teelken, 2012), bajo la creencia institucionalizada de que JCR es el indicador natural de calidad. Consecuentemente, el desequilibrio a favor de la función investigadora frente a las docente y de extensión no solo se acrecienta (Santos, 2009), sino que se abre una brecha interna dentro de esta función, aumentando la presión y las energías implicadas a la subfunción publicadora frente al resto de los cometidos y tareas de investigación, supeditadas a aquella. 
La importancia de la función docente se reduce no solo en términos relativos frente a la función investigadora, sino también en términos absolutos. Las sucesivas reformas y el nuevo imaginario académico transforma la función del docente hacia la de un gestor casi meramente administrativo de la capacidad de autoinstrucción del estudiante (Manzano-Arrondo y Andrés, 2007). La tecnificación progresiva de la actividad docente mediante plataformas de enseñanza virtual, la invasión de la organización mediante unidades de aprendizaje a través de diapositivas de Power-Point y similares, la fuerte burocratización a través de los protocolos de competencias y los formularios orientados a la evaluación de la calidad docente, entre otras prácticas, restan progresiva importancia a la creatividad, a la libertad de cátedra, o a la adaptación de la actividad a los acontecimientos de actualidad.

\section{Orientación economicista}

Manzano y Torrego (2009) describen tres modelos para concebir la orientación de la universidad. El modelo A puede ser denominado académico, y plantea el origen histórico como una comunidad de maestros y discípulos que construyen conocimiento desde el resultado del placer por saber, con fuertes exigencias de validez y honestidad científicas. El modelo B puede ser denominado economicista o mercantil; establece que la universidad ha de formar a profesionales de alto nivel requeridos para ingresar con éxito en el mercado laboral y construir conocimiento a la medida de las necesidades de los tejidos productivo y empresarial, adoptando además un enfoque de rendición de cuentas, eficiencia, subsidiaridad y productividad. El modelo $\mathrm{C}$ o de compromiso social plantea una clara orientación directa hacia el bien común, con altas exigencias de utilidad social en las tres funciones universitarias.

De las tres orientaciones, resulta evidente el protagonismo del modelo mercantil (De Witt, 2010), descrito desde términos como mercantilización (Susanti, 2011), privatización (Manzano-Arrondo, 2009), capitalismo académico (Johnson y Hirt, 2011), neoliberalismo universitario (Vidovich y Currie, 2011) o globalización neoliberal universitaria (Rizvi, 2006), entre otros.

Esta orientación ha sido denunciada y descrita en numerosas ocasiones (véase, por ejemplo, Almarcha, 2001; Codd, 2005; García, 2008; Johnson y Hirt, 2011; Manzano-Arrondo, 2009, 2011, 2012; ManzanoArrondo y Andrés, 2007; Shumar, 2004; Susanti, 2011; Teelken, 2012; Venieris y Cohen, 2004; Wareaas y Solbakk, 2009; Yamamoto, 2004). Rizvi (2006), por ejemplo, denuncia que el imaginario neoliberal en las políticas de educación mueve a la homogeneización internacional sin atender a las particularidades locales, según modelos hegemónicos. Partiendo de esta visión, los individuos son consumidores y la educación un bien privado.

El cuadro sintomático de esta fuerte orientación mercantil puede ser descrito sintéticamente en 17 puntos:

1. Reducción de la inversión económica estatal y aumento de la dependencia de fondos privados que orientan la docencia y la investigación hacia las necesidades del tejido empresarial.

2. Aumento de los costes de los estudios y recortes presupuestarios en profesorado, personal de administración y servicios, becas, 
proyectos de investigación e infraestructuras y medios materiales y humanos de apoyo.

3. Desaparición de titulaciones, centros e incluso universidades públicas; reubicación de profesorado, estudiantes y titulaciones.

4. Orientación de la actividad universitaria hacia la productividad cuantitativamente mensurable (número de egresos, publicaciones de impacto, nivel de calificaciones medias, fondos conseguidos, puestos en bibliotecas, etc.).

5. Enfoque homogeneizador e imposición de estándares no académicos para regular las actividades académicas; burocratización.

6. Estímulo de la competición entre estados, regiones, universidades, centros, departamentos, grupos de investigación, profesorado y estudiantado, en un mercado agresivo y altamente competitivo a nivel mundial.

7. Protagonismo del discurso de la élite, la aristocracia de los mejores, el mérito, el esfuerzo, la capacidad y el sacrificio, en sustitución del discurso sobre servicio público.

8. Fuerte aparición del branding universitario o inversión presupuestaria en marketing institucional orientado a la captación de clientes-estudiantes y contratos privados.

9. Sustitución de conceptos académicos por mercantiles: clientes, oferta, demanda, competitividad, eficiencia, déficit, mercado, coste cero, capitalización, liderazgo, excelencia, diferenciación, stakeholder, lobby, etc.

10. Virtualización de la enseñanza con una orientación homogeneizadora; aumento de la carga de trabajo al sumar la utilización de los medios informáticos a los presenciales (plataformas de enseñanza, correos electrónicos, foros, redes sociales, etc.).

11. Estímulo de la flexibilidad multidimensional autosufragada: movilidad geográfica, ductilidad de horarios, rotura de la distinción entre tiempos propios y laborales, instrucción continua, alta disponibilidad para cambiar de puesto u ocupación, etc.

12. Construcción de un imaginario de estrés continuo sin alternativa: modelo TINA (there is not alternative), metáfora del tren en marcha, vocabulario bélico (como luchar o morir), etc. Samuel Pisar (citado por Servan-Schreiber y Crecine, 1987) expresa muy claramente este imaginario cuando afirma que "la lógica del progreso sólo puede ser dominada por la excelencia (...). Los «campeonatos mundiales» de todas las categorías y de todos los campos nunca terminarán. No existen áreas protegidas. Todo el mundo debe jugar contra los campeones, lo quiera o no lo quiera, o convertirse en sus esclavos" (p. 90).

13. Naturalización de la creciente desigualdad generada por los puntos 6,7 y 12 , que construye un mundo de perdedores donde se salvan unos pocos ganadores, elegidos o excelentes.

14. Síndrome de la agenda completa: profesorado y estudiantes carecen de tiempo para la reflexión o la revolución, es decir, para ejercer una actitud crítica orientada hacia el diseño y construcción de alternativas. Sus agendas se orientan a responder a duras penas a las crecientes exigencias de valoración cuantitativa y a labores de autosalvación en un contexto hostil.

15. Desapasionamiento con la realización de las tareas universitarias, derivado principalmente de los puntos 3, 5, 10, 12 y 14 .

16. Conversión de la universidad en un espacio de negocio, que contrata y subcontrata, que interviene en el mercado como un 
consumidor de importancia creciente y ve sustituidas progresivamente más facetas (de organización, software, docencia, evaluación, mantenimiento, etc.) mediante agentes privados.

17. Sustitución del concepto de validez científica por el de calidad de gestión en el trono de la orientación académica. A su vez, reducción del concepto de calidad al de publicación en revistas indexadas en el JCR.

\section{Orientaciones liberadoras}

Para un sector creciente de la universidad, mayoritariamente perteneciente al estudiantado, resulta desconocido que la universidad puede ser y ha sido otra cosa. Frente al llamamiento por la construcción de un imaginario diferente (Rizvi, 2006), es importante también destacar que la educación superior pública ha sido tradicionalmente concebida como una institución social que sirve al bien común (Johnson y Hirt, 2011) y en cuyas cartas o documentos fundacionales consta de forma clara y explícita su orientación de servicio a la comunidad (Watson, 2008), sin mención expresa a ninguno de los protagonistas de los discursos actuales en términos de tejido productivo, mercado laboral, empresas, agentes de mercado o similares. El imaginario, por tanto, ya existe, era anterior al que dirige la actividad universitaria hoy. Y, es más, sigue siendo fuertemente defendido, sólidamente argumentado, imperiosamente necesario y perfectamente viable.

Hablamos de una educación superior política (Sousa, 2006), liberadora (Ellacuría, 1999), comprometida (Manzano-Arrondo, 2012) o construida desde la hélice emancipadora (Morin, Roger y Domingo, 2001). Ha sido defendida repetidas veces desde la máxima autoridad internacional en materia de educación, la UNESCO. En el acto específico para el diseño de la educación superior de mayor envergadura en la historia, concretado en París en 1998, tras años de trabajo de cientos de expertos y reuniones continentales, con la presencia de los máximos responsables políticos y académicos de las universidades del planeta, se llevó a cabo la Declaración Mundial de la Educación Superior (DMES). Fue una apuesta muy diferente a la que ese mismo año y en esa misma ciudad comenzó su andadura: el Espacio Europeo de Educación Superior, esta vez a manos de los máximos cargos políticos en materia de educación de los cuatro países europeos de economías más potentes y globalizadas: Reino Unido, Alemania, Francia e Italia, una semana después de que sus primeros ministros hubieran diseñado en el seno del G8 en Londres las directrices en materia universitaria que debían abundar en el mismo modelo de globalización economicista que fue presentado en sociedad en los ochenta.

Según el referente emancipador, liberador o de compromiso social, la educación superior ha de (UNESCO, 1998): promover aprendizaje, reflexión crítica, ciudadanía, desarrollo sostenible, mejora de la sociedad, igualdad, solidaridad y paz; a la vez que luchar contra la pobreza, la intolerancia, la violencia, el analfabetismo, el hambre, el deterioro del medio ambiente y las enfermedades. Cinco criterios orientan para ello (OREAL/UNESCO, 2007): trabajar en condiciones de igualdad (equidad), plantear objetivos que obedecen a toda la sociedad y no a grupos de poder (relevancia), garantizar resultados con significado para las personas en toda su diversidad (pertinencia), conseguir sus objetivos (eficacia) y hacerlo sin malgastar recursos (eficiencia). El re- 
sultado debería ser un conocimiento que (UNESCO, 2005): 1) se caracteriza por los objetivos y criterios mencionados; 2) va más allá del concepto «sociedad de la información», considerando expresamente las dimensiones social, ética y política; y 3) es compartido por toda la sociedad, lo que implica que no es utilizado por unos para el control, el aprovechamiento o la instrumentalización de otros.

La DMES, como el resto de los discursos para una educación superior socialmente comprometida, es difícilmente discutible. Por ello no es discutida. La orientación economicista hace frente a estos discursos principalmente mediante dos recursos. El primero, deplorado por Rodrigues (2007), uno de los promotores de la DMES, es el silencio. A pesar del llamamiento claro con que finalizaba la cita parisiense, instando a todos los Gobiernos del mundo a la construcción de la nueva universidad, lo cierto es que los responsables políticos habían apostado ya por la definición que días antes había establecido el grupo de los cuatro países europeos del G8. En contraste con la amplia difusión y el respaldo legislativo que han apoyado el diseño de La Sorbona, es difícil encontrar a miembros de la universidad que conozcan los términos de la DMES. El segundo recurso es la invasión del discurso economicista, definido en apartados anteriores.

\section{Unidad de Acción Comprometida}

Las Unidades de Acción Comprometida (UAC) constituyen una apuesta por diseñar y realizar prácticas universitarias plenamente instaladas en la orientación emancipadora, mostrando con ello la perfecta viabilidad de estos enfoques. Inicial y superficialmente presentadas en Manzano-Arrondo (2012), las UAC tienen la intención de construir prácticas (y los soportes normativos y estructurales que se requieren para hacerlas realidad) que pongan en funcionamiento las tres funciones de la universidad con igual protagonismo y una clara orientación directa hacia el Bien Común. Por Bien Común entendemos lo que Petrella (1997) define como "el conjunto de principios, reglas, instituciones y medios que permiten promover y garantizar la existencia de todos los miembros de una comunidad humana" (p. 18). No se trata únicamente de una existencia de subsistencia, sino de dignidad y decisiones compartidas (Belotti, 2014), sobre un asiento de moralidad democrática (Sanmartín, 2006). Una actividad, iniciativa o gestión orientada hacia el Bien Común, por tanto, implica en coherencia preocuparse por que los objetivos, los procedimientos y los resultandos fortalezcan ese conjunto de principios y se muestren en términos de mayor dignidad y existencia democrática.

Estos aspectos quedan en parte garantizados a partir de la participación del tercer sector, en el sentido de organizaciones de la sociedad civil sin ánimo de lucro, centradas en el servicio a la comunidad a través de valores de bien común como la solidaridad, el altruismo, la honestidad o la cooperación, y que representan un tercer poder frente al Estado y a los mercados (Rodríguez, 2005), con la clara intención de construir prácticas ciudadanas y democracia radical (Bel y Gómez, 2001).

Hasta la fecha se ha llevado a cabo una infinidad de experiencias UAC sin ser presentadas con esta denominación, sino concebidas desde dos referentes íntimamente relacionados: la investigación-acción participativa (IAP) y el aprendizaje-servicio (ApS).

La IAP se concibe desde la fusión de los cometidos de extensión 
(definido como cambio o transformación social) e investigación, realizadas simultáneamente en un trabajo horizontal con el tercer sector. Sin embargo, es muy habitual que tanto en la definición de su marco teórico como en su puesta en práctica participe directa o indirectamente el interés educador (Balcázar, 2003) e incluso ha sido defendida su localización natural en la práctica universitaria (Anderson, 2003) aunque siga siendo un referente básicamente desconocido en la educación superior (Brydon-Miller et al., 2003). Una UAC es una especie de IAP donde el cometido del aprendizaje forma parte fundacional del concepto y cuenta con la universidad como uno de los dos actores definitorios de la acción, a la vez que procura la presencia de varios sectores universitarios y varias disciplinas.

El ApS procura fundir en su fundación las funciones de extensión y docencia, entendidas respectivamente como servicio a la comunidad orientada a la transformación social y aprendizaje significativo y duradero (Coles, 2005). Igualmente, y como una consecuencia de su aplicación en el entorno universitario, es fácil encontrar referencias directas a que debe contar también con la investigación o construcción de conocimiento como una de sus columnas. Así, por ejemplo, Speck (2001) hace un llamamiento específico a reunir en prácticas de ApS las tres actividades universitarias, mientras que de forma análoga Butin (2006) habla de aprendizaje cognitivo y afectivo, investigación crítica y práctica reflexiva. Una UAC es una especie de ApS donde la construcción de conocimiento forma parte fundacional del concepto y cuenta con la universidad como uno de los dos actores definitorios de la acción, a

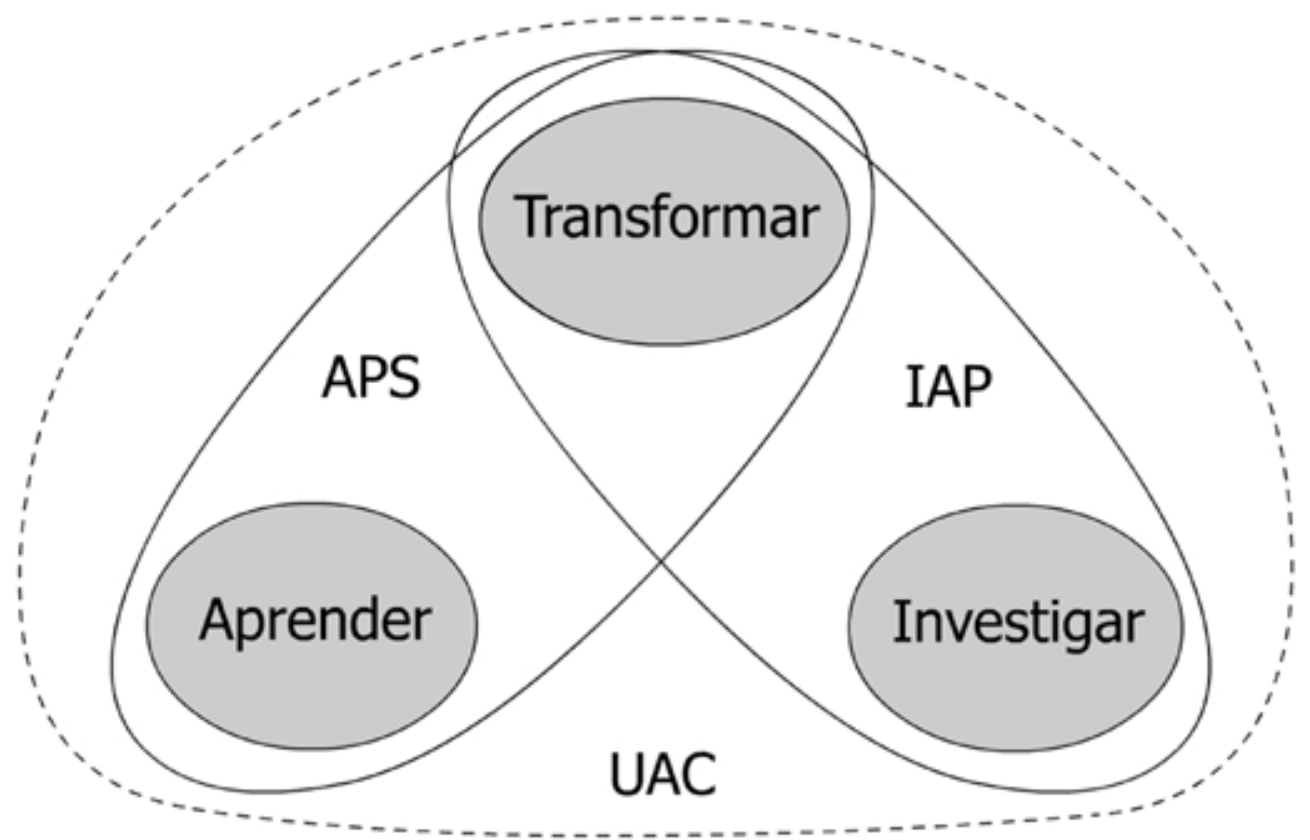

Figura 1. Ubicación de tres marcos de trabajo entre universidad y tercer sector. Fuente: elaboración propia. la vez que no se ciñe a los objetivos de aprendizaje desde el programa de una asignatura o unidad reglada. Incluso una titulación en su conjunto podría ser una UAC si cumple con sus características definitorias, englobando a las asignaturas, prácticas externas, trabajos fin de grado, etc., asociados a una problemática social o socioambiental concreta y una relación dialógica con el tercer sector.

En otras palabras, UAC se sitúa en acciones concretas susceptibles de ser consideradas como unidades coherentes, con sentido propio, y en donde la universidad, el

ApS y la IAP se confunden. La figura 1 intenta representar gráficamente los tres marcos de referencia mencionados.

Para que una referencia a UAC sea correcta es necesario que la práctica cumpla con una serie de características que pueden ser organizadas en cuatro elementos: qué, quién, cómo y cuándo. 


\subsection{Qué}

UAC arranca a partir de un reto, problema o necesidad social o socioambiental, bien sea de ámbito específico (acotado en el tiempo, el espacio y el grupo humano afectado) o general (funcionamientos, dinámicas o estructuras que construyen retos, problemas o necesidades específicas). El cuadro 1 muestra algunos ejemplos enlazados para los dos ámbitos.

\begin{tabular}{|l|l|}
\hline Específico & General \\
\hline $\begin{array}{l}\text { Privatización del servicio público del abastecimiento de } \\
\text { agua en un municipio concreto. }\end{array}$ & El agua como negocio frente al agua como derecho. \\
\hline $\begin{array}{l}\text { Desalojo de una comunidad campesina tras la recalifica- } \\
\text { ción de sus terrenos. }\end{array}$ & $\begin{array}{l}\text { Soberanía alimentaria, culturas campesina y urbana, hue- } \\
\text { lla ecológica y especulación urbanística. }\end{array}$ \\
\hline $\begin{array}{l}\text { Pauperización progresiva de un barrio de la periferia ur- } \\
\text { bana. }\end{array}$ & $\begin{array}{l}\text { Dinámicas de exclusión mediante prácticas urbanísticas, } \\
\text { mediáticas y legislativas. }\end{array}$ \\
\hline
\end{tabular}

\subsection{Quién}

Diferentes agentes coinciden con la misma intención de poner en juego sus especificidades para dar respuesta satisfactoria y coherente a ese qué. Para hablar con propiedad de una UAC, deben coparticipar al menos dos partes: universitaria y activista.

La parte activista está representada por una organización, asociación, plataforma o red del tercer sector, tal vez emergida como consecuencia del quéo con una trayectoria más amplia. La parte universitaria cuenta con al menos una unidad con sentido propio identificada claramente como parte de la universidad y que tal vez haya emergido también para este qué específico o partir de una trayectoria más amplia. Se trata de una asignatura concreta o un conjunto de ellas, de un título propio, de un trabajo fin de grado, de un programa de prácticas externas, de un movimiento social universitario... En cualquier caso, la aspiración es a incluir varios sectores (estudiantado, profesorado y personal de administración y servicios) y varias disciplinas, con la intención de incrementar la diversidad interna de la parte universitaria que forma parte de la unidad de acción.

El inicio del trabajo implica definir con la máxima claridad no solo el asunto que justifica el trabajo conjunto, sino la unidad de acción en concreto, es decir, la identidad colectiva sobre la que todo el equipo de trabajo se siente reflejado. Es una UAC porque existe unidad en la identidad del grupo. A partir de ese momento no es una plataforma ciudadana que intenta proteger un huerto urbano frente al nuevo trazado de una autovía, y que coincide con una parte universitaria interesada en comprender estos procesos y articular soluciones, sino que se trata de un mismo equipo de trabajo con una motivación común centrada en el qué y cuyo recorrido a partir de ese momento va a permitir conseguir objetivos diversos, definidos en el cómo.

\subsection{Cómo}

Si bien existe un qué perfectamente definido, ello es compatible con objetivos particulares no solo de la parte activista por su lado y la universitaria por el suyo, sino dentro de ambos grupos internamente. Esta
Cuadro 1. Ejemplos de ámbitos de trabajo de las UAC. Fuente: elaboración propia. 
realidad constatable en las experiencias IAP y ApS, no debe ser un obstáculo para la definición clara y operativa de tres objetivos, para un trabajo dialógico y para una actitud evaluadora.

Los tres objetivos de una UAC se corresponden con los tres cometidos característicos de la universidad. Ello insta a definir qué se pretende cambiar, qué se pretende aprender y qué conocimiento es el que se quiere construir (sistematizar y comunicar). Estas definiciones son comunes para toda la unidad, si bien, cada actor y parte añadirá las especificidades que considere. Así, por ejemplo, la parte universitaria debería contar con un interés especial por comprender los procesos que están en marcha, de dónde vienen y hacia dónde van, cotejándolos con otras situaciones y marcos teóricos, y generando con ello aprendizaje en todas las partes implicadas, así como sistematizando la información para someterla a procesos de comunicación. En cualquier caso, para hablar con propiedad de una UAC, no es viable que cada parte se reparta los objetivos. Es imprescindible que todos los actores cuenten con objetivos consensuados de acción, aprendizaje y construcción de conocimiento, explicitados y redactados al inicio del trabajo conjunto.

La dialogicidad o trabajo horizontal sin la participación de desequilibrios o procesos de dominación internos es una condición fundacional en el marco de la IAP, que se traslada literalmente a las UAC. Strier (2011), entre otros, llama la atención sobre el peligro que surge en las relaciones universidad-comunidad, debido al peso abrumador de los discursos académicos y a los medios habitualmente desiguales, de tal forma que se adoptan rápidamente desequilibrios en las dinámicas de trabajo.

La actitud evaluadora implica coherencia en los tres componentes ya mencionados (cognitivo, conativo y emotivo) en torno a la necesidad de evaluar dinámicas internas de trabajo, procesos de acción/ aprendizaje/construcción de conocimiento, y resultados parciales y finales. Para hacer posible estas prácticas es necesario explicitar previamente los estilos de trabajo y los puntos de llegada, sin cuyo auxilio es difícil llevar a cabo tareas de evaluación. No se trata de un aprobado/ suspendido al estilo académico, sino de dinámicas de reflexión y de conclusiones orientadas a mejorar el proceso en marcha y aprender de cara a procesos futuros.

Existe una amplia bibliografía en torno a los procesos de trabajo en ApS e IAP que son perfectamente trasladables al enfoque UAC, para concretar y singularizar un proceso temporalizado concreto. En Manzano-Arrondo (2014) puede consultarse el proceso exhaustivo en un ejemplo concreto de UAC, aplicado al proyecto y realización de un barómetro ciudadano. En el siguiente apartado se ejemplifica una aplicación concreta, realizada en un barrio de la ciudad, entre estudiantes de una asignatura, estudiantes de investigación en prácticas, profesorado universitario y una plataforma ciudadana.

\subsection{Cuándo}

La gestión del tiempo es uno de los frentes más difíciles de solucionar en cualquier proyecto donde coexistan tercer sector y universidad. Sus agendas llevan ritmos muy diferentes, porque así lo exigen sus respectivas funciones específicas.

La universidad se encuentra fuertemente mediatizada por la actividad docente y la investigadora. La actividad docente se articula en años 
académicos, en tramos de curso entero o de medio curso. Si la UAC se establece en torno a una asignatura, la duración de esta condiciona las posibilidades de sinergia entre ambas partes, obligando a definir un trabajo conjunto acotado en un curso completo o en medio curso. La actividad investigadora tiene flujos más abiertos, pero igualmente se encuentra sujeta a tramos habituales de uno a cuatro años, en función del proyecto. Los trabajos fin de carrera y de máster son actividades básicamente de investigación, pero con ritmos de docencia. Las tesis doctorales permiten mucho más juego, si bien igualmente están sujetas a plazos.

El tercer sector combina dos fuentes de ritmo: el reto y los huecos de activismo. La principal fuente es el reto o problema que justifica la existencia de la organización. Si se trata de un ámbito específico, los flujos de trabajo son difícilmente predecibles y habitualmente se muestran condensados y exigen agilidad en la reacción. Una plataforma ciudadana que intenta evitar la privatización del servicio municipal de aguas, reacciona según los pasos que vaya dando el Gobierno local, sus declaraciones en prensa o las convocatorias de licitación, por ejemplo. En ocasiones, hay que reaccionar de una día para otro. Estos retos obligan a un nivel de compromiso más elevado por parte de la academia y constituyen los focos de necesidad a la vez más urgentes y de más difícil sinergia. Los problemas de ámbito general son mucho más sencillos de abordar para los ritmos y herramientas de la universidad. Es relativamente sencillo acotar subobjetivos dentro del reto general y abordarlos según los ritmos académicos. Los huecos de activismo constituyen otra fuente de posibles inconvenientes en la gestión del tiempo. La población activista no es profesional del activismo. Con suerte, sus miembros tienen trabajos diversos que imponen sus ritmos sobre los tiempos de ocio que estas personas han decidido dedicar a la mejora de la sociedad. Esta circunstancia añade fragilidad a los retos temporales. Las reuniones no pueden ser continuas y habitualmente se ajustan a periodos que no tienen por qué coincidir con la parte universitaria. Es fácil que siempre falten miembros concretos por motivos laborales o bien porque no pueden postergar más los asuntos personales que se han visto atrasados debido a la opción del activismo. Suelen ser personas muy saturadas de tareas, y, por tanto, poco dispuestas a asumir más cargas, derivadas de la colaboración con la universidad. Es imprescindible tener en cuenta que la colaboración será posible solo si los miembros de la universidad juegan al mismo juego, es decir, asumen que han de implicar también parte de sus tiempos de ocio para resolver satisfactoriamente los retos comunes. El trabajo conjunto funcionará tanto mejor cuanto la universidad transite también por los huecos de activismo.

\section{Una aplicación en un contexto de barrio}

En la ciudad de Sevilla se encuentra Tres Barrios, una de las Zonas Necesitadas de Transformación Social (ZNTS) de Andalucía, es decir, un sector poblacional y urbano que sufre problemas de exclusión presente y amenazas de empeoramiento futuro. Está constituido por varias barriadas de origen obrero y del extra-radio, que finalmente han sido absorbidas por el crecimiento urbano. Cuenta con viviendas mayoritariamente sin bajos comerciales, sin ascensor, de pequeñas dimensiones, con población envejecida, inmigrantes, gitanos y altos niveles de 
paro, entre otros aspectos. A su vez, se trata de una zona históricamente muy bien articulada en torno a organizaciones del tercer sector, muchas de las cuales se agruparon hace pocos años en una plataforma ciudadana muy activa.

En lo que sigue, exponemos una síntesis de la experiencia, sin ánimo de exhaustividad sino con la intención de mostrar una concreción UAC, especialmente en lo tocante al diseño y el proceso.

\subsection{Qué}

Algunos miembros de la plataforma tenían ya experiencia de relación con miembros de la universidad en proyectos diversos, fácilmente definibles de IAP, mientras que otros han surgido desde la perspectiva de ApS. En 2013, el colectivo ciudadano llama a uno de los activistas universitarios con los que ha trabajado, para poner en marcha una investigación sobre los valores de la población juvenil del barrio que permita diseñar una intervención posterior. Los miembros de la plataforma observan que los adolescentes del barrio reproducen casi fielmente los valores transmitidos en unas pocas series televisivas, cuentan con dificultades de empatía y el peligro de perpetuar la situación de marginalidad en que han crecido.

El equipo de trabajo acota el objetivo en la población de estudiantes de Enseñanza Secundaria Obligatoria (15-16 años), que cursan en los cuatro institutos donde asisten los jóvenes del barrio, durante el mes de marzo de 2014, aplicando un cuestionario ad hoc basado en experiencias previas de medición de valores en juventud. Los resultados del estudio constituirán la materia prima para diseñar una intervención posterior en el barrio y en los institutos entrevistados.

\subsection{Quién}

Con ese objetivo en mente, se construye una sinergia que implica a:

- Estudiantes provenientes de dos grupos de clase de una asignatura de primer curso de grado. El objetivo curricular de la asignatura es básicamente el aprendizaje del análisis de datos. Se plantea adquirir los conocimientos asociados a ese objetivo académico dando respuestas al objetivo especificado para el equipo UAC. Ello implica recoger los datos de los institutos, introducirlos en ordenador, realizar los análisis y elaborar resultados que los estudiantes presentarán en un foro comunitario en el barrio. Dado que la capacidad de absorber grupos de estudiantes es limitada en esta UAC, del número de candidatos se realiza una selección al azar, hasta completar las 25 plazas ofertadas. El resto de los estudiantes de ambos grupos de clase cubren sus objetivos académicos en otro frente de trabajo en la ciudad.

- El profesor responsable de esta asignatura y estos dos grupos de clase, con experiencia de relación con la plataforma ciudadana desde los tres cursos académicos previos.

- Una estudiante de prácticas de investigación que además es vecina de la zona.

- Un estudiante que realiza su trabajo fin de grado sobre esta experiencia.

- La plataforma ciudadana de Tres Barrios que trabaja sobre asuntos de multiculturalidad e integración en la zona. Dentro de esta 
plataforma, la organización que se implica directamente en el proyecto cuenta con profesorado de institutos de enseñanza secundaria y activistas relacionados con la juventud y la inmigración.

\subsection{Cómo}

El recorrido previo común con que cuentan los miembros de la plataforma y el profesor universitario permite no abordar una primera fase de gran importancia: implicar esfuerzo y tiempo específicos para generar conocimiento mutuo y confianza. El primer paso real en esta implementación lo constituye clarificar el objetivo central e identificar algunas barreras que amenazan un buen desarrollo del proyecto:

- Coparticipan estudiantes con recorridos vitales y experiencias muy dispares, de los que se sospecha motivaciones muy diferentes para organizar su tiempo.

- Algunos participantes (la casi totalidad de los estudiantes universitarios implicados en ambos grupos de clase) carecen de experiencia directa o indirecta en campos de trabajo en contextos reales y retos sociales. Solo una estudiante de los 25 que participan en esta experiencia, tiene alguna experiencia activista.

- Los institutos se encuentran saturados. Al formar parte de una zona marginada, son fruto de visitas continuas por parte de investigadores diversos que dificultan en cierta medida el desarrollo normal de las actividades y que no suelen dejar una huella positiva ni útil. Esta práctica continuada ha generado resistencia para recibir a equipos de investigación.

Todos los agentes participantes responden a un cuestionario o a una entrevista personal con la intención de clarificar qué esperan aprender, transformar y sistematizar/comunicar. Se pone en marcha una comisión específica que tiene en cuenta estas respuestas para, sin necesidad de organizar más reuniones, matizar todo el proceso para posibilitar que todo el mundo consiga sus objetivos específicos a lo largo de la experiencia y las especificidades aporten riqueza al conjunto.

Si bien todos los agentes tienen la oportunidad de dejar su impronta en el diseño y realización de la experiencia, en la práctica tiene lugar un reparto específico de tareas: el diseño general de la experiencia corre a cargo de la plataforma, el profesor y el estudiante fin de grado; el cuestionario es diseñado por el profesor y revisado por la plataforma; el trabajo de campo es diseñado y supervisado por el profesor y la estudiante en prácticas de investigación; la recogida de datos y su introducción al ordenador es realizada por los estudiantes de primer curso; los análisis de datos los realiza el profesor, mientras que la estudiante en prácticas de investigación utiliza este informe técnico para orientar el aprendizaje práctico de los estudiantes de primer curso; el diseño del foro comunitario se lleva a cabo entre la plataforma, la estudiante en prácticas de investigación y el profesor; en su realización participan todos los agentes; y el informe final es redactado por el estudiante de fin de grado y el profesor.

Los resultados son expuestos en un foro comunitario, es decir, una reunión en la que es convocada la vecindad y en la que asisten, además de habitantes del barrio, todos los agentes que han participado en el proceso. En el foro, se exponen los resultados y se abre un debate 
con el público, que culmina en una mesa redonda específica, establecimiento de conclusiones y un acto reivindicativo simbólico en la plaza principal del barrio, donde se lee un manifiesto.

\subsection{Cuándo}

La gestión de la agenda muestra más inconvenientes de los que estaban previstos en un primer momento. El proceso temporal diseñado implicaba: 1) formar y seleccionar los grupos de estudiantes que se dedicarían a la realización de las entrevistas; 2) concertar los momentos de entrevista con los institutos; 3) recoger, introducir y procesar los datos; 4) organizar la presentación de los resultados y los materiales de apoyo; y 5$)$ realizar el foro.

No obstante, los problemas de tiempo y la saturación de los institutos retardaron los momentos viables para realizar la recogida de datos. Hubo que vencer varias resistencias, comprensibles desde la experiencia previa de estos centros. El papel de la estudiante en prácticas de investigación resultó fundamental para vencer esta desconfianza inicial y finalmente todos los institutos participaron en la experiencia, si bien aproximadamente con un mes de retraso frente al cronograma inicial. Este retraso obligó a forzar las siguientes fases, exigiendo un sobreesfuerzo para todos los agentes implicados.

\section{Discusión}

Frente a los problemas de desajuste, desequilibrio y orientación economicista de las tres funciones básicas de la universidad, hemos planteado la propuesta de las Unidades de Acción Comprometida como una contribución específica al conjunto de soluciones que se están articulando sobre el mismo tema.

La implementación de una UAC acumula los mismos problemas que han venido ya describiéndose en las aplicaciones de IAP y ApS, a la vez que suma los derivados de su implementación en un contexto de orientación mercantil de la educación superior, descrita en el cuadro sintomático expuesto en un apartado anterior y que apunta a diversos inconvenientes:

1. UAC no es una realidad visible, no se ha constituido como un referente accesible en el contexto universitario, fuertemente adoctrinado en el academicismo. La pureza del conocimiento científico no incluye la urgencia de los retos sociales y, por el contrario, se instala en una versión de neutralidad y objetividad con amplia tradición en la universidad (Holsteller, 1992) y en los sistemas educativos (Yogev y Michaeli, 2011).

2. UAC requiere creatividad, valentía e inicialmente más tiempo de lo que suele exigir la inercia. La relativa lentitud y falta de control sobre la situación genera miedo en la academia, habituada a procesos más ágiles y artificiales (Fox). En un contexto de agenda completa, UAC no tendrá éxito.

3. UAC queda de momento fuera de los estándares burocratizados en la medición de la calidad y la excelencia, por lo que su elevada utilidad pública y académica cuenta con dificultades de concreción. 
La especificidad de las UAC es orientar la potencia de la ciencia y de la academia para organizar unidades de aprendizaje y transformación social en sinergia con el tercer sector. Tal especificidad implica niveles de exigencia superiores a los que puedan encontrarse en los estándares de calidad al uso (Manzano-Arrondo, 2012), puesto que los requerimientos reales necesitan de implementaciones con el mínimo de fisuras. A su vez, aumenta la utilidad pública de las instituciones de educación superior y permite una formación mejor instalada en la complejidad de los contextos reales. Sin embargo, a pesar de estas ventajas, los importantes inconvenientes hacen pronosticar que las UAC no van a ser implementadas a medio plazo salvo por miembros de la academia y de las organizaciones capaces de implicar dosis de coraje y valentía suficientes como para ir modificando el imaginario académico y transformando los contraproducentes criterios de medición de calidad que sufre hoy la universidad. Una vez finalizada esta fase de construcción de caminos, contaremos en el viaje con quienes se suman únicamente a los caminos ya trazados. 


\section{Referencias bibliográficas}

Almarcha, A. (2001). Misión de la universidad. Ensenanza superior y competitividad: la globalización de los mercados, Revista Española de Investigaciones Sociológicas, 93, p. 205-220.

Anderson, S. G. (2003). Engaging students in community-based research: A model for teaching social work research, Journal of Community Practice, 10, p. 71-87.

Andrés, L. y Manzano-Arrondo, V. (2004). ¿Hacia dónde camina la universidad? Reflexiones en torno al EEES. Revista Interuniversitaria de Formación del Profesorado, 18, p. 269-276.

Balcazar, F. E. (2003). Investigación acción participativa (iap): Aspectos conceptuales y dificultades de implementación. Fundamentos en Humanidades, 4, p. 59-77.

Bel, C. y Gómez, J. (2001). El tercer sector a debate. Papeles de Geografía, 33, p. 35-47.

Belotti, F. (2014). Entre el bien común y el buen vivir. Afinidades a distancia. Íconos. Revista de Ciencias Sociales, 48, p. 41-54.

Brydon-Miller, M.; Greenwood, D. y Maguire, P. (2003) Why action research? Action Research, 1, 1, p. 9-28.

Butin, D.W. (2006). The limits of service-learning in Higher Education. The Review of Higher Education, 29, p. 473-498.

Cerezo, H. (2007) ¿Publicar o morir? Elementos, 66, p. 21-25.

Cheng, M. (2001). The perceived impact of quality audit on the work of academics. Higher Education Research Eं Development, 30, p. 179-191.

Christensen, T. (2011). University governance reforms: potential problems of more autonomy?. Higher Education, 62, p. 503-517.

Clarck, B. (1983). The Higher Education System: Academic organisations in crossnational perspective. Berkeley: University of California Press.

Codd, J. (2005). Teachers as 'managed professionals' in the global education industry: the New Zealand experience. Educational Review, 57, p. 193-206.

Coles, E. A. (2005). Why do Service-Learning? Issues for first-time faculty. En Bellner, M. y Pomery, J. (Eds.), Service-Learning: Intercommunity $\mathcal{E}$ Interdisciplinary Explorations. Indianopolis: University of Indianopolis Press, 2005, p. 85-95.

Corlett, J.A. (2005). The good professor. Journal of Academic Ethics, 3, p. 27-54.

Cunningham, F. (2007). The university and social justice. Journal of Academic Ethics, 5, p. 153-162.

Dawes, R. M. (1975). Fundamentos y técnicas de medición de actitudes. México: Limusa.

De Wit, K. (2010). The networked university: the structure, culture, and policy of universities in a chan- ging environment. Tertiary Education and Management, 16, p. 1-14.

Derrida, J. (2002). Universidad sin condición. Madrid: Trotta.

Ellacuria, I. (1999). Escritos Universitarios. San Salvador: UCA Editores.

Festinger, L. (1957). The Theory of Cognitive Dissonance. Stanford: Stanford University Press.

Fox, R. (2004). To be academic or not to be academic. Journal of Community E Applied Social Psychology, 14, p. 302-304.

García-Quero, F. (2014). Crisis y Universidad: de intelectuales a hacedores de 'papers'. eldiario. es, 30 de junio de 2014. Recuperado el 10 de julio de 2014 de: http:/ / www.eldiario.es/zonacritica/Crisis-Universidad-intelectuales-hacedores-papers_6_265683463.html

García, M.J. (2008). El impacto de la globalización en la universidad del siglo XXI. Tendencias Pedagógicas, 13 , p. 59-78.

Greenwood, D.J. (2012). Doing and learning action research in the neo-liberal world of contemporary higher education. Action Research, 10, 2, p. 115-132.

Guillén, M. (2003). Hacia una revisión crítica del análisis neoclásico del consumo: una alternativa basada en las necesidades. Revista de Economía Crítica, 1, p. 95-111.

Hosteller, K. (1991). Community and neutrality in critical thought: a nonobjetivist view on the conduct and teaching in critical thinking. Educational Theory, 41, p. 1-12.

Johnson, A. T. y Hirt, J. B. (2011). Reshaping academic capitalism to meet development priorities: the case of public universities in Kenya. Higher Education, 61, p. 483-499.

Macfarlane, B. (2011). Professors and intellectual leaders: formation, identity and role. Studies in Higher Education, 36, p. 57-73.

Manzano-Arrondo, V. (2011). El papel de la universidad en la sociedad de ignorantes. Científica, 12, p. 29-55.

—. (2014). Il Barómetro Cittadino. La risposta dell'Universitá per unire didattica, ricerca, azione. Rivista Internazionale di EDAFORUM, 9, p. 23. Recuperado el 10 de julio de 2014 de: http:/ / rivista.edaforum.it/numero23/monografico_Manzano.html . (2012). La Universidad Comprometida. Vitoria: Hegoa.

- (2009). Qué cosa es esa de la universidad privatizada. Opciones Pedagógicas, 39, p. 112-124.

Manzano-Arrondo, V. y Andrés, L. (2007). El diseño de la nueva universidad europea. Algunas causas, algunas consecuencias. Sevilla: Atrapasueños. 
Manzano-Arrondo, V. y Torrego, L. (2009). Tres modelos para la universidad. Revista de Educación, 350, p.477-489.

Martínez, J. (2012). Enseñar en la universidad pública. Sujeto, conocimiento y poder en la Educación Superior. Revista Aula de Encuentro, Núm. especial, p. 39-52.

Morales, G. (2004). La universidad pública y el campo académico universitario: propuesta de análisis cultural. Recuperado el 10 de julio de 2014 de: http:/ / www. fcs.una.ac.cr/doc_fcs/launiversidadcomocampodeestudio.pdf

Morales, P. (2000). Medición de actitudes en psicología y educación. Madrid: Universidad Pontificia de Comillas.

Morin, E., Roger, E. y Domingo, R. (2001). Educar en la era planetaria. El pensamiento complejo como Método de aprendizaje en el error y la incertidumbre humana. Valladolid: Universidad de Valladolid.

Morrison, E.; Rudd, E.; Picciano, J. y Nerad, M. (2011). Are you satisfied? PhD education and faculty taste for prestige: limits of the prestige value system. Research of Higher Education, 52, p. 24-46.

OREAL/UNESCO (2007). El derecho a una educación de calidad para todos en América Latina y el Caribe. Revista Electrónica Iberoamericana sobre Calidad, Eficacia y Cambio en Educación, 5, 3, p. 1-21.

Petrella, R. (1997). El Bien Común. Elogio de la solidaridad. Madrid: Debate,.

Prilleltensky, I. (2008). The role of power in wellness, oppression, and liberation: The promise of psychopolitical validity. Journal of Community Psychology, 36, p. 116-136.

(2004). Validez psicopolítica: el próximo reto para la psicología comunitaria. En Montero, M. (Comps.), Introducción a la psicología comunitaria. Desarrollo, conceptos y procesos. Buenos Aires: Paidós, p. 13-32.

Quinn, L. (2012). Understanding resistance: an analysis of discourses in academic staff development. Studies in Higher Education, 37, p. 69-83.

Rizvi, F. (2006). Imagination and the globalisation of educational policy research. Globalisation, Societies and Education, 4, p. 193-205.

Rrodrigues, M.A. (2007). El regional y el global: la articulación de la diversidad de funciones de la enseñanza superior. Revista de Universidad y Sociedad del Conocimiento, 4, p. 5-15.

Rodríguez, J. (2005). Tercer sector: una aproximación al debate sobre el término. Revista de Ciencias Sociales, 11, 3, p. 464-474.

Sanmartín, J. (2006). El bien común como idea política. John Stuart Mill, los liberales y sus críticos. Foro Interno, 6, p. 125-153.

Santos, M.A. (2009). Introducción. En M.A. Santos (Eds.), Politicas educativas y compromiso social. El pro- greso de la equidad y la calidad. Madrid: Octaedro Editorial, p. 13-22.

Servan-Schreiber, J. y Crecine, B. (1987). La revolución del conocimiento. Barcelona: Plaza \& Janés.

Shumar, W. (2004). Global pressures, local reactions: higher education and neo-liberal economic policies. International Journal of Qualitative Studies in Education, 17, p. 823-839.

Siegel, B. L. (1984). Knowledge with Commitment: teaching is the central task of the university. Vital Speeches of the Day, 50, 13, p. 394-397.

Sousa, B. (2006). La universidad popular del siglo XXI. Lima: Fondo Editorial de la Facultad de Ciencias Sociales.

Speck, Br. W. (2001). Why Service-Learning? New Directions for Higher Education, 114, p. 3-13.

Strier, R. (2011). The construction of university-community partnerships: entangled perspectives. Higher Education, 62, p. 81-97.

Susanti, D. (2011). Privatisation and marketisation of higher education in Indonesia: the challenge for equal access and academic values. Higher Education, 62, p. 209-218.

Teelken, Ch. (2012). Compliance or pragmatism: how do academics deal with managerialism in higher education? A comparative study in three countries. Studies in Higher Education, 37, p. 271-290.

UNESCO (1998). Conferencia Mundial sobre la Educación Superior. La educación superior en el siglo XXI. Visión y acción. París: Ediciones de la UNESCO.

UNESCO (2005). Hacia las sociedades del conocimiento. París: Ediciones de la UNESCO.

Venieris, G. y Cohen, S. (2004). Accounting reforms in Greek universities: a slow moving process. Financial Accountability E Management, 20, p. 183-204.

Vidovich, L. y Currie, J. (2011). Governance and trust in Higher Education. Studies in Higher Education, 36, p. 43-56.

Wareaas, A. y Solbakk, M. N. (2009). Defining the essence of a university: lessons from higher education branding. Higher Education, 57, p. 449-462.

Watson, D. (2008). The university in the modern word. Ten lessons of civic and community engagement. Education, Citizenship and Social Justice, 3, p. 43-55.

Worthington, A. C. y Higgs, H. (2011). Economies of scale scope in Australian higher education. Higher Education, 62, p. 387-414.

Yamamoto, K. (2004). Corporatization of national universities in Japan: revolution for governance or rhetoric for downsizing? Financial Accountability $\mathcal{E}^{\mathcal{O}} \mathrm{Ma}$ nagement, 20, p. 153-181.

Yogev, E. y Michaeli, N. (2011). Teachers as society-involved "organic intellectuals": training teachers in a political context. Journal of Teacher Education, 62, p. 312-324. 
MANZANO-ARRONDO, Vicente y SUÁREZ GARCÍA, Enrique. Unidad de acción comprometida: una propuesta de solución ante el problema universitario del servicio a la sociedad. Hábitat y Sociedad, 2015, n. ${ }^{\circ} 8$, pp. 147-165.

$<$ www.habitatysociedad.us.es>

http://dx.doi.org/10.12795/HabitatySociedad.2015.i8.07

○ 